\title{
Equidistribution for Sequences of Polynomial Mappings
}

\section{Alexander Russakovskit Mikhail Sodin}

Abstract. We consider sequences of polynomial mappings $P_{k}$ : $\mathbb{C}^{n} \rightarrow \mathbb{P}^{m}$ with increasing degrees $d_{k}=\operatorname{deg} P_{k}$ and prove that

(i) preimages of most of the complex hyperplanes in $\mathbb{P}^{m}$ are equidistributed with the pullbacks $P_{k}^{*} \omega$ of the standard invariant (FubiniStudy) form $\omega$ on $\mathbb{P}^{m}$;

(ii) if all $P_{k}$ are non-degenerate, then preimages of most of the points in $\mathbb{P}^{m}$ are equidistributed with $P_{k}^{*} \omega^{m}(m \leq n)$.

0. Introduction. This paper was inspired by a number of recent papers on holomorphic dynamics in several complex variables [BS1, BS2, HP, FS], where various versions of the theorem on equidistribution of preimages under iterations were proved.

We remind the statement of this remarkable theorem, which was proved by $\mathrm{H}$. Brolin $[\mathrm{Br}]$ for polynomials and by $\mathrm{M}$. Lyubich $[\mathrm{L}]$ and independently by A. Freire, A. Lopes and R. Mañé [FLM] for rational functions:

Let $R(z)$ be a rational function of degree $d \geq 2$, and let $R^{n}$ be its $n$-th iteration. Then for all $a \in \mathbb{P} \backslash \mathcal{E}_{R}, \operatorname{card}\left(\mathcal{E}_{R}\right) \leq 2$,

$$
\frac{1}{d^{n}}\left(R^{n}\right)^{*} \delta_{a} \rightarrow \mu
$$

Here $\left(R^{n}\right)^{*} \delta_{a}$ is a discrete measure in $\mathbb{P}$, counting the number of roots of the equation $R^{n}(z)=a$ with multiplicities; convergence is in the weak sense. 
Later on, a proof of (0.1) based on value distribution theory was given in [ES]. Systematic application of potential theory methods in this circle of problems was initiated by N. Sibony.

The limit measure $\mu$ in (0.1) possesses a number of properties which are very important in dynamical applications (see the survey [EL], Ch. 3).

If $R$ is a polynomial, this measure appears to be the equilibrium measure of the Julia set $J_{R}$.

It turns out that a similar phenomenon occurs in some other situations in fields different from dynamics. We mention below one example from polynomial approximation (the result is essentially due to H.-P. Blatt and E. Saff):

Let $f(z)$ be a continuous function on a (non-polar regular) compact set $K \subset \mathbb{C}$, analytic in the interior of $K$ and not extendible analytically through any point of $\partial K$. Let $P_{n}$ be the sequence of polynomials of best uniform approximation to $f$ on $K$. Then for some subsequence $n_{k} \rightarrow \infty$ and all $a \in \mathbb{C}$,

$$
\frac{1}{n_{k}} P_{n_{k}}^{*} \delta_{a} \rightarrow \mu,
$$

where $\mu$ is the equilibrium measure of the set $K$.

There arises a natural question: What happens for an arbitrary given sequence of polynomials no matter where it originates from?

In the paper [So], an analogue of $(0.1)-\left(0.1^{\prime}\right)$ was proved for arbitrary sequences of rational functions $\mathcal{R}=\left\{R_{n}\right\}$ with increasing degrees $d_{n}{ }^{1}$ :

For all $a \in \mathbb{P} \backslash \mathcal{E}(\mathcal{R})$,

$$
\frac{1}{d_{n}}\left(R_{n}\right)^{*}\left(\delta_{a}-\omega\right) \rightarrow 0
$$

Here $R_{n}^{*} \omega$ is the pullback of the spherical area element, and the exceptional set $\mathcal{E}(\mathcal{R})$ has Hausdorff dimension zero. The atomic measure $\delta_{a}$ may be replaced in $(0.2)$ by an arbitrary probability measure in $\mathbb{P}$, which does not charge points of $\mathcal{E}(\mathcal{R})$.

It turned out that the faster the sequence of degrees $d_{n}=\operatorname{deg}\left(R_{n}\right)$ grows, the smaller the exceptional set $\mathcal{E}(\mathcal{R})$ is. Thus, for example, when $\sum 1 / d_{n}<\infty$, the set $\mathcal{E}(\mathcal{R})$ is polar. Besides that, for each increasing function $\varphi(t), \varphi(0)=0$, one may find such an increasing sequence of positive integers $\delta_{n}$ that for each sequence of rational functions $\mathcal{R}=\left\{R_{n}\right\}, \operatorname{deg} R_{n} \geq \delta_{n}$, the Hausdorff $\varphi$-measure of the exceptional set $\mathcal{E}(\mathcal{R})$ equals zero.

The present paper appeared as an effort to understand multidimensional analogues of the results of [So].

\footnotetext{
${ }^{1}$ Accidentally, there are disappointing misprints in the statements of main results of [So] while all the proofs are correct.
} 
1. Main Results. Below we introduce some definitions and state principal results. We use standard notations $d=\partial+\bar{\partial}, d^{c}=(4 \pi i)^{-1}(\partial-\bar{\partial})$. Points in $\mathbb{P}^{m}$ are denoted by capital letters $Z, W, \ldots$ and identified with their representation in homogeneous coordinates $Z=\left[Z_{0}, Z_{1}, \ldots, Z_{m}\right]$. Points in $\mathbb{C}^{n}$ are denoted by small letters $z, w, \ldots$ By $\omega$ we denote the Fubini - Study form $\omega=d d^{c} \log |Z|^{2}$ on $\mathbb{P}^{m} ; \omega_{0}$ is its affine analogue $\omega_{0}=d d^{c} \log \left(1+|z|^{2}\right)$.

Our main tool is potential theory methods. Since $\mathbb{P}^{m}$ is compact, there exist no plurisubharmonic functions on it. However, because of the local nature of all our assertions, it is enough to consider "plurisubharmonic sections" of the trivial linear fibre bundle over $\mathbb{P}^{m}$. This means that on each standard chart $\Omega_{j}=\left\{Z: Z_{j} \neq 0\right\}$ there is a plurisubharmonic function $u_{j}$, and on $\Omega_{i} \cap \Omega_{j}$ the difference $u_{i}-u_{j}$ is the pluriharmonic function $\log \left|Z_{j}\right|^{2} /\left|Z_{i}\right|^{2}$. Hence the current $T$ given by $T=d d^{c} u_{j}$ on $\Omega_{j}$ is globally well defined. Similarly the difference of two plurisubharmonic sections is a globally defined function on $\mathbb{P}^{m}$.

Let $\mathcal{P}=\left\{P_{k}\right\}$ be a sequence of polynomial mappings $\mathbb{C}^{n} \rightarrow \mathbb{P}^{m}$. Denote by $\operatorname{deg} P_{k}$ the maximum of degrees of the components of $P_{k}$. We call a sequence $\mathcal{P}$ lacunar if $\sum 1 / d_{k}<\infty$ where $d_{k}=\operatorname{deg} P_{k}$.

Any hyperplane $\mathcal{W}$ in $\mathbb{P}^{m}$ may be represented in the form

$$
\mathcal{W}=\left\{Z:\langle W, Z\rangle \stackrel{\text { def }}{=} \sum_{j=0}^{m} W_{j} Z_{j}=0\right\}
$$

where $W \in \mathbb{P}^{m}$ corresponds to $\mathcal{W}$. Hence the set of all hyperplanes is naturally parametrized by points of $\mathbb{P}^{m}$. Studying preimages of hyperplanes under the sequence $P_{k}$ is the same as studying zeros of the sequence of linear combinations $\left\langle W, P_{k}(z)\right\rangle$, which are polynomials in $z \in \mathbb{C}^{n}$, with respect to the parameter $W \in \mathbb{P}^{m}$.

The current of integration over the preimage of $\mathcal{W}$ with respect to a mapping $P$ is denoted by $\left[P^{-1} \mathcal{W}\right]$. By $P^{*} \omega$ we denote the pullback by $P$ of the FubiniStudy form $\omega$. Since this form is smooth, its pullback is well defined. We preserve the same denotion for forms and for currents induced by these forms. Pullbacks of currents are discussed later on.

Now, by analogy with Nevanlinna Theory (see e. g. [Sh]) we define "the proximity sequence" for a given hyperplane $\mathcal{W}$ :

$$
m_{k}(\mathcal{W})=m(k, \mathcal{W}, \mathcal{P})=\int_{\mathbb{C}^{n}} \log \frac{|W|^{2}\left|P_{k}(z)\right|^{2}}{\left|\left\langle W, P_{k}(z)\right\rangle\right|^{2}} \omega_{0}^{n}(z),
$$

and the set of exceptional hyperplanes

$$
\mathcal{E}(\mathcal{P})=\left\{\mathcal{W}: \limsup _{k \rightarrow \infty} \frac{m_{k}(\mathcal{W})}{d_{k}}>0\right\}
$$

is a natural analogue of the set of Valiron deficiencies. 
Remark 1.1 It may happen that the mappings $P_{k}$ are degenerate, e.g., if $P_{k}\left(\mathbb{C}^{n}\right)$ is contained in a hyperplane $\mathcal{W}_{0}$. Then $m_{k}\left(\mathcal{W}_{0}\right)$ is $+\infty$ for all $k$, and $\mathcal{W}_{0}$ is included in the set $\mathcal{E}(\mathcal{P})$.

Remark 1.2 We will often identify the hyperplane $\mathcal{W}$ with its representative (normal vector) $W$ in the parameter space when there is no danger of misunderstanding. For instance, $m_{k}(\mathcal{W})$ and $m_{k}(W)$ will actually mean the same.

We say that sequences of $(p, p)$-currents $\mathcal{A}_{k}$ and $\mathcal{B}_{k}$ are $d_{k}$-equidistributed if

$$
\frac{1}{d_{k}^{p}}\left(\mathcal{A}_{k}-\mathcal{B}_{k}\right) \rightarrow 0, k \rightarrow \infty .
$$

in the sense of currents.

Theorem 1.1 Let $\mathcal{P}$ be a sequence of polynomial mappings with increasing degrees. Then:

(1) for every non-exceptional hyperplane $\mathcal{W}$ the sequences of $(1,1)$-currents $\left[P_{k}^{-1} \mathcal{W}\right]$ and $P_{k}^{*} \omega$ are $d_{k}$-equidistributed;

(2) the set $\mathcal{E}(\mathcal{P})$ of exceptional hyperplanes has Hausdorff dimension not exceeding $2 m-2$ in the parameter space $\mathbb{P}^{m}$;

(3) if the sequence $\mathcal{P}$ is lacunar, then $\mathcal{E}(\mathcal{P})$ is pluripolar in $\mathbb{P}^{m}$.

At this point, we note that in several variables the value distribution theory studies preimages of various analytic sets in the image space. When the image space is $\mathbb{P}^{m}$, all the analytic sets are algebraic, i.e., defined by systems of homogeneous polynomial equations in $\mathbb{P}^{m}$. Given a homogeneous polynomial mapping $Q: \mathbb{P}^{m} \rightarrow \mathbb{C}^{q}, 0 \leq q \leq m$, one may consider the analytic set $Q^{-1}(0)$ and study its preimages under sequences of polynomial mappings into $\mathbb{P}^{m}$. Hyperplanes are just the simplest particular case where $q=1$ and $Q$ is just a linear polynomial $\langle W, Z\rangle$. A natural object to study in general is the behavior of sequences of solutions of systems of polynomial equations

$$
Q\left(P_{k}(z)\right)=0
$$

with respect to $Q$. Certainly, the set of polynomial mappings $Q$ must be parametrized, i.e., these polynomials must be considered as points of some certain space. When $q=1$ or $q=m$ and the degree of $Q$ is 1 , the parameter space is just $\mathbb{P}^{m}$ itself.

As it will be shown in Section 4, Theorem 1.1 may be formally strengthened. Hyperplanes may be replaced by algebraic divisors, or, equivalently, linear combinations may be replaced by homogeneous polynomials. Moreover, coefficients of those polynomials may themselves be polynomials in $z$. Besides that, the exceptional set of a lacunar sequence is in fact pluripolar in each local analytic 
variety of positive dimension which is not contained in any hyperplane. The precise formulation of the result may be found in Theorem 4.1 (Section 4).

To formulate Theorem 1.2, introduce some notations. Let $W \in \mathbb{P}^{m}$. One can consider $W$ as the intersection point of $m$ hyperplanes in $\mathbb{P}^{m}$. It is convenient to choose these hyperplanes in the following way.

Suppose that $W$ lies in the chart $\Omega_{0}$. Then $W=\left[1, w_{1}, \ldots, w_{m}\right]$ and we choose the normal vectors of the hyperplanes as follows:

$$
W^{(1)}=\left[-w_{1}, 1,0, \ldots, 0\right], \ldots, W^{(m)}=\left[-w_{m}, 0, \ldots, 0,1\right]
$$

(modifications for other charts are obvious).

Define a positive $(m-1, m-1)$-form $\Lambda_{W}(Z)$ by

$$
\Lambda_{W}(Z)=g_{W} \sum_{j=0}^{m-1}\left(\omega-d d^{c} g_{W}\right)^{j} \wedge \omega^{m-j-1},
$$

where

$$
g_{W}(Z)=\log \frac{m|Z|^{2}|W|^{2}}{\sum\left|\left\langle W^{(j)}, Z\right\rangle\right|^{2}} .
$$

If the point $W$ lies in the chart $\Omega_{0}$, and hence has the form $[1, w], w \in \mathbb{C}^{m}$, then for $Z=[1, z] \in \Omega_{0}$ we have

$$
\left\langle W^{(j)}, Z\right\rangle=z_{j}-w_{j}
$$

and hence

$$
\Lambda_{W}=\log \frac{m\left(1+|z|^{2}\right)\left(1+|w|^{2}\right)}{|z-w|^{2}} \sum_{j=0}^{m-1}\left(d d^{c} \log |z-w|^{2}\right)^{j} \wedge \omega^{m-1-j} .
$$

This implies that the form $\Lambda_{W}$ is a smooth form on $\mathbb{P}^{m} \backslash W$ and has an integrable singularity of order $\log r / r^{2 m-2}$ at the point $W$. Therefore it represents a positive current. The form $\Lambda_{W}$ is standard in value distribution theory (compare [Sh], Ch. 2). It actually originates from the Poincaré-Lelong formula for vector functions.

It is useful to outline more explicitly the character of dependence of $\Lambda_{W}(Z)$ on the parameter $W$. Let $A_{j}$ be the $(m+1) \times(m+1)$-matrix with elements $a_{n m}, a_{0 j}=-1, a_{j 0}=1, j=1, \ldots, m$, and $a_{i j}=0$ for all other pairs of $(i, j)$. Then for $W \in \Omega_{0}$ and $W^{(j)}$ chosen as above, we can rewrite $\left\langle W^{(j)}, Z\right\rangle$ in the form $W A_{j} Z$ ( $W$ is considered here as a row vector and $Z$ as a column vector). Thus one can write

$$
\begin{aligned}
\Lambda_{W}(Z)=\log \frac{m|Z|^{2}|W|^{2}}{\sum\left|W A_{j} Z\right|^{2}} \sum_{j=0}^{m-1}\left(d d^{c} \log \sum\left|W A_{j} Z\right|^{2}\right)^{j} & \wedge \omega^{m-1-j}(Z),
\end{aligned}
$$


which implies homogeneity in $W$.

In what follows we will need to define pullbacks of certain currents with respect to polynomial mappings. This procedure requires certain explanations. They will be given in Section 3 .

Until the end of this section we assume that $n \geq m$. We will call a mapping $P: \mathbb{C}^{n} \rightarrow \mathbb{P}^{m}$ non-degenerate if the image of $\mathbb{C}^{n}$ has non-empty interior in at least one local chart of $\mathbb{P}^{m}$. This means that the Jacobian matrix of our mapping has rank $m$ outside of some analytic set $X$. As it will be explained in Section 3, the pullback of $\Lambda_{W}$ by $P$ is well-defined for $W \notin P(X)$.

Consider a sequence of non-degenerate mappings $P_{k}: \mathbb{C}^{n} \rightarrow \mathbb{P}^{m}$. Let $X_{k}$ be the set where the rank of $J_{P_{k}}$ is less than $m$. If $W \notin P_{k}\left(X_{k}\right)$, which is a pluripolar set, the current $P_{k}^{*} \Lambda_{W}$ is well defined. Let $Y=\bigcup_{k} P_{k}\left(X_{k}\right)$. In view of all the above, the whole sequence of currents $P_{k}^{*} \Lambda_{W}$ is well defined outside of the pluripolar set $Y$.

The "proximity sequence" and the set of exceptional points are defined as follows:

$$
\begin{gathered}
m_{k}(W)=m(k, W, \mathcal{P})= \begin{cases}\int_{\mathbb{C}^{n}} P_{k}^{*} \Lambda_{W} \wedge \omega_{0}^{n-m+1}(z), & \text { if } W \notin P_{k}\left(X_{k}\right), \\
m_{k}(W)=+\infty & \text { otherwise; }\end{cases} \\
\mathcal{E}(\mathcal{P})=\left\{W: \limsup _{k \rightarrow \infty} \frac{m_{k}(W)}{d_{k}^{m}}>0\right\} .
\end{gathered}
$$

Theorem 1.2 Let $\mathcal{P}$ be a sequence of non-degenerate polynomial mappings $\mathbb{C}^{n} \rightarrow \mathbb{P}^{m}, n \geq m$, with increasing degrees. Then:

(1) for every non-exceptional point $W$ the sequences of $(m, m)$-currents $P_{k}^{-1} W$ and $P_{k}^{*} \omega^{m}$ are $d_{k}$ - equidistributed;

(2) the set $\mathcal{E}(\mathcal{P})$ of exceptional points has Hausdorff dimension not exceeding $2 m-2$ in the parameter space $\mathbb{P}^{m}$;

(3) if the sequence $\mathcal{P}$ is lacunar, then the sequences $P_{k}^{-1} W$ and $P_{k}^{*} \omega^{m}$ are $d_{k}$ equidistributed outside of a pluripolar set in $\mathbb{P}^{m}$.

Remark 1.3 We were able to prove that the set $\mathcal{E}(\mathcal{P})$ is pluripolar only in the equidimensional case $n=m$. The proof, as well as analogues of Theorems 1.1-1.2 for intermediate codimensions, will be published elsewhere.

Remark 1.4 Note that Theorem 1.2 provides non-trivial information about distribution of preimages of points only if the sequence $1 / d_{k}^{m} P_{k}^{*} \omega^{m}$ has non-zero limit points.

Remark 1.5 All our results are also valid for polynomial mappings $\mathbb{C}^{n} \rightarrow$ $\mathbb{C}^{m}$. It is enough to add $P_{0}=1$ to the mapping $\left(P_{1}, \ldots, P_{m}\right)$ to obtain a mapping into $\mathbb{P}^{m}$ and apply Theorems 1 and 2 . 
The rest of the paper is organized as follows. In Section 2 we give elementary examples illustrating the results; in Section 3 some background on currents, pluripotential theory and value distribution theory is provided for reader's convenience. It contains also the proofs of some auxilliary results. In Section 4 we prove Theorem 1.1 and several variations of it; Section 5 contains the proof of Theorem 1.2.

2. Examples. Consider a simple example: Let $P_{k}(z)=\left(z_{1}^{d_{k}}, z_{2}^{d_{k}}\right)$ be mappings $\mathbb{C}^{2} \rightarrow \mathbb{C}^{2}$ with $d_{k} \nearrow \infty$ (equivalently, we may consider mappings $P_{k}(z)=\left[1, z_{1}^{d_{k}}, z_{2}^{d_{k}}\right]$ into $\left.\mathbb{P}^{2}\right)$. It is easy to see that the uniform convergence $\left(1 / d_{k}\right) \log \left(1+\left|P_{k}(z)\right|^{2}\right) \rightarrow G(z)$ takes place, where

$$
G(z)=\sup \left(\log ^{+}\left|z_{1}\right|^{2}, \log ^{+}\left|z_{2}\right|^{2}\right)
$$

is the plurisubharmonic Green function of the unit polydisk with pole at infinity. It follows that

$$
d d^{c} G=\lim _{k \rightarrow \infty} \frac{1}{d_{k}} P_{k}^{*} \omega
$$

The current $d d^{c} G$ is concentrated on the boundary of the unit polydisk and on the set $\left\{z:\left|z_{1}\right|=\left|z_{2}\right|>1\right\}$.

Consider preimages of hyperplanes under this sequence of mappings. Theorem 1.1 says that most of them should "converge to the carrier of $d d^{c} G$ ". Elementary geometric considerations allow us to find the exceptional hyperplanes.

Every hyperplane of the form $z_{1}=c$ or $z_{2}=c$ has preimages of the form $z_{1}=c^{\prime}\left(z_{2}=c^{\prime}\right)$. These preimages tend to concentrate to the cylinder $\left|z_{1}\right|=1$ (respectively $\left|z_{2}\right|=1$ ). Thus all such hyperplanes are exceptional. A third family of exceptional hyperplanes consists of those passing through the origin. In the parameter space, exceptional hyperplanes correspond to points forming three hyperplanes in $\mathbb{P}^{2}:\left\{W_{0}=0\right\},\left\{W_{1}=0\right\},\left\{W_{2}=0\right\}$. So the exceptional set has dimension $2=2 m-2$.

Now consider preimages of points. The current $\left(d d^{c} G\right)^{2}$, being the limit of $\left(1 / d_{k}^{2}\right) P_{k}^{*} \omega^{2}$, is concentrated on the distinguished boundary of the unit polydisk; hence preimages of most points must tend to the torus by theorem 2. However, all points of the form $(0, c)$ or $(c, 0)$ have preimages of the form $\left(0, c^{\prime}\right)$ (respectively $\left.\left(c^{\prime}, 0\right)\right)$ and are definitely exceptional. We have again an exceptional set of dimension $2 m-2$.

Remark 2.1 The example above shows that even in the case of iterations of a polynomial mapping $\left(d_{k}=d^{k}\right)$ it is not possible to have a smaller exceptional set than in the general situation. There remains a question, whether in $\mathbb{P}^{m}$ the exceptional set really "looks like" $m+1$ hyperplanes.

3. Auxilliary Results. In this section we discuss the basic concepts of the theory of currents, the pluripotential theory and the value distribution theory and prove a couple of auxilliary statements. 
The reader is referred for instance to the books $[\mathrm{LG}],[\mathrm{Kl}],[\mathrm{Sh}]$. We also use some ideas of L. Gruman [Gr].

3.1. Currents. We use the standard notation $\mathcal{C}^{k}$ for the space of functions whose $k$-th derivative is continuous, $\mathcal{C}_{0}^{k}$ stands for the set of $\mathcal{C}^{k}$-functions with compact support and $\mathcal{D}=\mathcal{C}_{0}^{\infty}$. The space $\mathcal{D}$ is endowed with the topology of uniform convergence of the functions and all derivatives. The elements of the dual space, $\mathcal{D}^{\prime}$ are called distributions.

Let $\varphi$ be a differential form of degree $(p, q)$ with $\mathcal{C}_{0}^{\infty}$ coefficients:

$$
\begin{gathered}
\varphi=\sum_{|I|=p,|J|=q} \varphi_{I J} d z_{I} \wedge d \bar{z}_{J}, \\
I=\left(i_{1}, \ldots, i_{p}\right), \quad J=\left(j_{1}, \ldots, j_{q}\right), \quad d z_{I}=d z_{i_{1}} \wedge \ldots \wedge d z_{i_{p}}, \quad \varphi_{I J} \in \mathcal{C}_{0}^{\infty} .
\end{gathered}
$$

The space of all such forms (usually called test forms) is denoted by $\mathcal{D}_{p, q}$. This space is endowed with the topology of uniform convergence of coefficients and all their derivatives. Currents of degree $(p, q)$ in $\mathbb{C}^{n}$ are defined as continuous linear functionals on $\mathcal{D}_{n-p, n-q}$. The currents of degree $(n, n)$ are thus distributions.

Let $\beta=\left(d d^{c}|z|^{2}\right)^{n}$ be the Euclidean volume measure in $\mathbb{C}^{n}$. A differential form $\varphi \in \mathcal{C}_{(p, p)}^{\infty}$ is called positive if for every system $\alpha_{1}, \ldots, \alpha_{n-p}$ of complex linear forms (which means $\alpha_{i}=\sum_{j=1}^{n} a_{i j} d z_{j}, a_{i j} \in \mathbb{C}$ ) the product

$$
\varphi \wedge i \alpha_{1} \wedge \overline{\alpha_{1}} \wedge \ldots \wedge i \alpha_{n-p} \wedge \bar{\alpha}_{n-p}=\psi \beta
$$

is such that $\psi \geq 0$.

A current $T \in \mathcal{D}_{n-p, n-p}^{\prime}$ is called positive if for every system $\alpha_{1}, \ldots, \alpha_{p}$ of complex linear forms with constant coefficients and every non-negative test function $\varphi \in \mathcal{D}$,

$$
T\left(\varphi i \alpha_{1} \wedge \overline{\alpha_{1}} \wedge \ldots \wedge i \alpha_{p} \wedge \bar{\alpha}_{p}\right) \geq 0
$$

As one easily sees, positive forms induce positive currents via integration:

$$
(\psi, \varphi)=\int_{\mathbb{C}^{n}} \psi \wedge \varphi .
$$

Positive currents were introduced and studied by P. Lelong.

If a current can be extended to a continuous linear functional on the space $\mathcal{C}_{0,(p, q)}^{0}$ of continuous $(p, q)$-forms with compact support, then it is said to be continuous of order zero. If a current $T \in \mathcal{D}_{0,0}^{\prime}$ is continuous of order zero, then it is a (complex) measure.

A positive current $T \in \mathcal{D}_{p, p}^{\prime}$ can be written in the form

$$
T=i^{n-p} \sum_{|I|=n-p,|J|=n-p} T_{I J} d z_{I} \wedge d \bar{z}_{J}
$$


and the distributions $\mu_{I J}=T_{I J} \beta$ are then complex measures (and $\mu_{I I}$ are positive measures).

One can define derivatives of a current $T \in \mathcal{D}_{p, q}^{\prime}$ according to the formulae

$$
\begin{aligned}
& \partial T(\varphi)=(-1)^{p+q}(T, \partial \varphi) \\
& \bar{\partial} T(\varphi)=(-1)^{p+q}(T, \bar{\partial} \varphi) .
\end{aligned}
$$

If $d T=0$, the current is called closed. The most important examples of positive closed currents are currents of integration over an analytic variety $X$ (denoted by $[X]$ ) and the currents of the form $d d^{c} V$ with $V$ plurisubharmonic.

For $\varphi \in \mathcal{D}_{p, q}$, denote by $\|\varphi\|$ the quantity

$$
\|\varphi\|=\sup |\varphi|(z), \quad|\varphi|=\sup _{I, J}\left|\varphi_{I J}(z)\right| .
$$

The mass norm of a current $T$ is a measure defined as

$$
M_{T}(\Omega)=\sup \{|(T, \varphi)|:\|\varphi\| \leq 1, \operatorname{supp} \varphi \subset \Omega\} .
$$

Suppose that a current $T$ is defined outside of some analytic set $X$ and one would like to extend it over $X$. If such an extension exists and has zero mass on $X$, it is called simple. If the current $T$ is continuous of order zero, its simple extension over $X$ exists if $T$ has locally bounded mass in the neighborhood of $X$. A theorem of H. Skoda [Sk] (see also [LG], Theorem 2.44) says that if $T$ is a closed positive current with locally finite mass in a neighborhood of $X$, its simple extension over $X$ is a positive closed current in $\mathbb{C}^{n}$.

Let $A$ and $B$ be complex manifolds of dimension $n$ and $m$, respectively, and let $P: A \rightarrow B$ be a holomorphic mapping with Jacobian matrix of maximal rank. Let $T$ be a current of degree $(p, q)$ on $B$ and let $\varphi \in \mathcal{D}_{n-p, n-q}(A)$ be a test form. The pullback of $T$ is a current $P^{*} T$ on $A$ acting as follows:

$$
\left(P^{*} T, \varphi\right)_{A}=\left(T, \int_{P(x)=y} \varphi(x)\right)_{B}
$$

for a test form $\varphi(x) \in C_{0}^{\infty}(A)$. Here multiplicities (Lelong numbers) of the fibers $P^{-1} y$ are taken into account (if $T$ is represented by a smooth form, this all is just equivalent to the change of variables formula).

If the mapping $P$ is proper one can define also the pushforward of a current. Let $T$ be a current of degree $(n-p, n-q)$ on $A$ and let $\varphi \in \mathcal{D}_{p, q}(B)$ be a test form. The pushforward of $T$ is the current $P_{*} T$ of degree $(m-p, m-q)$ on $B$ defined by

$$
\left(P_{*} T, \varphi\right)_{B}=\left(T, P^{*} \varphi\right)_{A},
$$

where $P^{*} \varphi$ is the form obtained by substituting $y=P(x)$ in $\varphi$ and expressing all the differentials in terms of $x$ and $d x$. 
In the definitions above, $p$ and $q$ are supposed to be integers between 0 and $\min (n, m)$ so that the corresponding denotions make sense. The operators $P_{*}$ and $P^{*}$ commute with differentiation.

For our purposes we would like to define pullbacks of certain currents in the case when the mapping $P$ does not have Jacobian matrix of maximal rank. A mapping $P: \mathbb{C}^{n} \rightarrow \mathbb{P}^{m}$ is called non-degenerate if the image of $\mathbb{C}^{n}$ has non-empty interior in at least one local chart of $\mathbb{P}^{m}$. This means that the Jacobian matrix of our mapping has rank $m$ outside of some analytic set $X$.

Hence one can define $P^{*} T$ in $\mathbb{C}^{n} \backslash X$ and the problem is to decide when it is extendible onto all of $\mathbb{C}^{n}$. By the Skoda theorem mentioned above, this problem is reduced to checking that $P^{*} T$ has bounded mass near $X$. Before we discuss this problem further, it is useful to review certain notions of the pluripotential theory.

\subsection{Plurisubharmonic functions and Monge-Ampère operatorsAs} we have mentioned already, if $v$ is a plurisubharmonic function then the $(1,1)-$ current $T=d d^{c} V$ is a positive closed current. One would like to define $\left(d d^{c} v\right)^{2}$ or, more generally, $d d^{c} u \wedge d d^{c} v$ for $u$ and $v$ plurisubharmonic. Both $d d^{c} u$ and $d d^{c} v$ have measure coefficients but measures cannot be multiplied in general. However, if $u$ is locally bounded, the current $u d d^{c} v$ is well defined, and we define $d d^{c} u \wedge d d^{c} v$ by

$$
d d^{c} u \wedge d d^{c} v=d d^{c}\left(u d d^{c} v\right)
$$

The wedge product $d d^{c} u \wedge d d^{c} v$ is again a closed positive current. Given locally bounded plurisubharmonic functions $u_{1}, \ldots, u_{p}$ we define inductively

$$
d d^{c} u_{1} \wedge \ldots \wedge d d^{c} u_{p}=d d^{c}\left(u_{1} d d^{c} u_{2} \wedge \ldots \wedge d d^{c} u_{p}\right)
$$

The operators $u \mapsto\left(d d^{c} u\right)^{p}$ are called Monge-Ampère operators. For $p>1$ they are not linear and not continuous in general. However, they are continuous with respect to (locally) uniformly convergent and decreasing pointwise convergent sequences of plurisubharmonic functions.

In certain situations it is possible to define the products $d d^{c} u \wedge T, T$ a closed current, $u$ plurisubharmonic, also in the case when $u$ is not bounded below everywhere, especially when $u$ has logarithmic singularities. We refer to the paper [De] for such results.

The simplest case (nevertheless providing the general idea) is $u_{w}=$ $\log |z-w|^{2}$. Let $\alpha_{w}=d d_{z}^{c} u_{w}$. The form $\alpha_{w}$ is positive, closed and smooth in $\mathbb{C}^{n} \backslash\{w\}$. Hence there is no difficulty to define $\alpha_{w}^{p}$ in $\mathbb{C}^{n} \backslash\{w\}$. It is straightforward to check that the mass of $\alpha_{w}^{p}$ is bounded in the neighborhood of $w$ for $p<n$, and therefore its simple extension exists. When $p=n$, the current still extends to a current in the entire $\mathbb{C}^{n}$; in fact, $\alpha_{w}^{n}=0$ in $\mathbb{C}^{n} \backslash\{w\}$ and the Poincaré-Lelong formula implies that $\alpha_{w}^{n}=\delta_{w}$. 
To ensure a somewhat deeper insight of the mentioned facts, we would like to cite a couple of statements from Demailly's paper [De], where an extensive exposition is given.

Following [De], the unbounded locus $L(u)$ of a plurisubharmonic function $u(z)$ is defined as the set of such points $z$ that $u$ is unbounded in every neighborhood of $z$.

Theorem 3.1 ([De], Theorem 2.5) Let $u_{1}, \ldots, u_{q}$ be plurisubharmonic functions and let $T$ be an $(n-p, n-p)$-current, $p>0$. The currents

$$
u_{1} d d^{c} u_{2} \wedge \cdots \wedge d d^{c} u_{q} \wedge T
$$

and

$$
d d^{c} u_{1} \wedge d d^{c} u_{2} \wedge \cdots \wedge d d^{c} u_{q} \wedge T
$$

are well defined and have locally finite mass as soon as $q \leq p$ and the sets

$$
L\left(u_{j_{1}}\right) \bigcap \cdots \bigcap L\left(u_{j_{m}}\right) \bigcap \operatorname{supp} T
$$

have Hausdorff $(2 p-2 m+1)$-measure zero for all choices of indices $j_{1}<\ldots<j_{m}$ in $\{1, \ldots, q\}$.

We emphasize a particular case.

Corollary 3.1 ([De], Corollary 2.11) Let $u_{1}, \ldots, u_{q}$ be plurisubharmonic functions such that $L\left(u_{j}\right)$ is contained in an analytic set $A_{j}$ for every $j$. Then $d d^{c} u_{1} \wedge \ldots \wedge d d^{c} u_{q}$ is well defined provided

$$
\operatorname{codim}\left(A_{j_{1}} \cap \ldots \cap A_{j_{m}}\right) \geq m
$$

for all choices of indices $j_{1}<\ldots<j_{m}$ in $\{1, \ldots, q\}$.

Corollary 3.2 If $u$ is plurisubharmonic in $\mathbb{C}^{n}$ and $L(u)$ is an analytic set, then the current $\left(d d^{c} u\right)^{q}$ is well defined when $\operatorname{dim} L(u) \leq n-q$.

For $u_{w}$ the unbounded locus $L\left(u_{w}\right)$ is just $w$ and hence the currents $u_{w} \alpha_{w}^{q-1}$ and $\alpha_{w}^{q}$ are well defined for all $q$.

Consider the singular form $\Lambda_{w}$ defined by (1.4). In our definitions,

$$
\Lambda_{w}(z)=\left(\log m+\log \left(1+|z|^{2}\right)+\log \left(1+|w|^{2}\right)-u_{w}\right) \sum_{j=0}^{m-1} \alpha_{w}^{j} \wedge \omega^{m-1-j},
$$

so it is a linear combination of the terms of the form $u_{w} \alpha_{w}^{j} \wedge \omega^{m-1-j}$ or $v \alpha_{w}^{j} \wedge$ $\omega^{m-1-j}, v$ a smooth function. In view of what has been mentioned above, $\Lambda_{w}$ is well defined as a current in $\mathbb{C}^{m}$ and has locally finite mass.

Let $P: \mathbb{C}^{n} \rightarrow \mathbb{P}^{m}, n \geq m$, be a non-degenerate mapping and let $X$ be the set where the rank of $J_{P}$ is less than $m$. We would like to define the pullback 
of $\Lambda_{W}$ by $P$ for $W \notin P(X)$. Consider the functions $U_{w}=u_{w} \circ P$ on $\mathbb{C}^{n}$. Then $L\left(U_{w}\right)=P^{-1}(w)$. If $w \notin P(X)$, then $\operatorname{dim} L\left(U_{w}\right)=n-m$ and therefore, by the corollary above, $\Lambda_{W} \circ P$ is well defined and has locally finite mass everywhere in $\mathbb{C}^{n}$. Since on $\mathbb{C}^{n} \backslash X$ the mapping $P$ has maximal rank, the current $P^{*} \Lambda_{W}$ is well defined and coincides with $\Lambda_{W} \circ P$. Since it has locally finite mass everywhere, according to Skoda's Theorem it extends over $X$ to a current in the whole $\mathbb{C}^{n}$, which we shall still denote by $P^{*} \Lambda_{W}$.

In fact, it is not necessary to use such strong means as Demailly's Theorem to justify the pullback of $\Lambda_{W}$. Since $\Lambda_{W}$ is a smooth form in $\mathbb{P}^{m} \backslash W$ its pullback is well defined outside of $P^{-1} W$. If $W \notin P(X)$, then $P^{-1} W \bigcap X=\varnothing$ and hence $P^{*} \Lambda_{W}$ extends over $X$. It remains to check that it extends over $P^{-1} W$ but, again, since $P^{-1} W \cap X=\varnothing$, the form $P^{*} \Lambda_{W}$ is integrable near $P^{-1} W$ and hence has locally finite mass.

3.3. Plurisubhamonic functions of logarithmic growth. In the sequel we often use the names $\mathcal{L}$ and $\mathcal{L}_{+}$for the classes of plurisubharmonic functions $u(z)$ satisfying respectively the relations $u(z) \leq \log \left(1+|z|^{2}\right)+O(1)$ and $u(z)=$ $\log \left(1+|z|^{2}\right)+O(1)$ on $\mathbb{C}^{n}$. We define $\mathcal{L}\left(\mathbb{P}^{m}\right)$ and $\mathcal{L}_{+}\left(\mathbb{P}^{m}\right)$ to be the sets of plurisubharmonic sections of the trivial fibre bundle over $\mathbb{P}^{m}$ belonging to $\mathcal{L}$ (respectively $\mathcal{L}_{+}$) in each standard chart of $\mathbb{P}^{m}$. In other words, if $u_{j}$ is the representative of the section in the chart $\Omega_{j}$, then $u_{j} \leq \log \left(|Z|^{2} /\left|Z_{j}\right|^{2}\right)+O(1)$ $\left(u_{j}=\log \left(|Z|^{2} /\left|Z_{j}\right|^{2}\right)+O(1)\right.$ respectively $)$.

Functions from $\mathcal{L}_{+}$are locally bounded by definition. Hence, if $u_{j} \in \mathcal{L}_{+}\left(\mathbb{C}^{n}\right)$, $j=1, \ldots, n$, the currents $d d^{c} u_{1} \wedge \cdots \wedge d d^{c} u_{n}$ are well defined.

We would like to use a result of B. A. Taylor $[\mathrm{T}]$.

Lemma 3.3 ([T]) Let $u$ and $v$ be locally bounded plurisubharmonic functions in $\mathbb{C}^{n}$, such that $v>0, v \rightarrow \infty$ when $|z| \rightarrow \infty$ and

$$
u(z) \leq v(z)+o(v(z)), \quad|z| \rightarrow \infty
$$

Then:

$$
\int_{\mathbb{C}^{n}}\left(d d^{c} u\right)^{n} \leq \int_{\mathbb{C}^{n}}\left(d d^{c} v\right)^{n} .
$$

In particular, if

$$
u(z)=v(z)+o(v(z)), \quad|z| \rightarrow \infty
$$

then

$$
\int_{\mathbb{C}^{n}}\left(d d^{c} u\right)^{n}=\int_{\mathbb{C}^{n}}\left(d d^{c} v\right)^{n} .
$$


Remark 3.4 As noted in $[\mathrm{T}]$, the lemma holds for $\left(d d^{c} u\right)^{n}$ replaced by $\left(d d^{c} u_{1}\right)^{k} \wedge\left(d d^{c} u_{2}\right)^{n-k}$ (or even $d d^{c} u_{1} \wedge \cdots \wedge d d^{c} u_{n}$ ) and the right-hand side is multiplied by some constant $k_{n}$ depending only on $n$, provided all the $u_{j}$ satisfy (3.1). To see this, apply the lemma to $u=x u_{1}+y u_{2}$ and $(x+y) v, x, y \geq 0$, expand $\left(d d^{c}\left[x u_{1}+y u_{2}\right]\right)^{n}$ and $(x+y)^{n}\left(d d^{c} v\right)^{n}$, and compare terms.

Taking $v(z)=\log \left(1+|z|^{2}\right)$ and using the fact that

$$
\int_{\mathbb{C}^{n}}\left(d d^{c} v\right)^{n}=\int_{\mathbb{C}^{n}} \omega^{n}=1,
$$

we see that if locally bounded functions $u_{i}, i=1, \ldots, n$, belong to $\mathcal{L}_{+}$, then

$$
\int_{\mathbb{C}^{n}} d d^{c} u_{1} \wedge \cdots \wedge d d^{c} u_{n}=1
$$

and if they belong to $\mathcal{L}$, then

$$
\int_{\mathbb{C}^{n}} d d^{c} u_{1} \wedge \cdots \wedge d d^{c} u_{n} \leq k_{n} .
$$

Actually the proof of the lemma in $[\mathrm{T}]$ mentioned above contains a stronger statement (though not explicitly formulated): the function $u(z)$ need not be locally bounded; it is enough to have $\left(d d^{c} u\right)^{n}$ well-defined and to put no mass on the unbounded locus $L(u)$ (with obvious modifications in the case of several functions).

For the reader's convenience we reproduce the proof here. For $\varepsilon>0, C>0$ consider the function $w(z)=(1+\varepsilon) v(z)-C$ and the set $G=G(\varepsilon, C)=\{z \in$ $\left.\mathbb{C}^{n}: w(z)<u(z)\right\}$. The set $G$ is relatively compact in $\mathbb{C}^{n}$ since $\varepsilon>0$, and $\mathrm{u}(\mathrm{z})$ is locally bounded on $G$. Hence one can apply the comparison theorem of Bedford and Taylor ([BT], Theorem 4.1) to obtain

$$
\int_{G}\left(d d^{c} u\right)^{n} \leq \int_{G}\left(d d^{c} w\right)^{n}=(1+\varepsilon)^{n} \int_{G}\left(d d^{c} v\right)^{n} \leq(1+\varepsilon)^{n} \int_{\mathbb{C}^{n}}\left(d d^{c} v\right)^{n} .
$$

If one lets $C \rightarrow \infty$, then it follows that

$$
\int_{\mathbb{C}^{n} \backslash L(u)}\left(d d^{c} u\right)^{n} \leq(1+\varepsilon)^{n} \int_{\mathbb{C}^{n}}\left(d d^{c} v\right)^{n} .
$$

So if $\left(d d^{c} u\right)^{n}$ puts no mass on $L(u)$, then

$$
\int_{\mathbb{C}^{n}}\left(d d^{c} u\right)^{n} \leq(1+\varepsilon)^{n} \int_{\mathbb{C}^{n}}\left(d d^{c} v\right)^{n}
$$

and letting $\varepsilon \rightarrow 0$, one obtains (3.2). 
If $u_{1}$ and $u_{2}$ are two functions satisfying (3.1) we can apply the same reasoning to $u=\left(u_{1}+u_{2}\right) / 2$, and $(3.6)$ implies in this case that

$$
\int_{\mathbb{C}^{n} \backslash\left\{L\left(u_{1}\right) \cup L\left(u_{2}\right)\right\}}\left(d d^{c} u_{1}\right)^{j} \wedge\left(d d^{c} u_{2}\right)^{n-j} \leq k_{n} \int_{\mathbb{C}^{n}}\left(d d^{c} v\right)^{n} .
$$

Note that if $u \in \mathcal{L}\left(\mathbb{P}^{m}\right)$ and $P: \mathbb{C}^{n} \rightarrow \mathbb{P}^{m}$ is a polynomial mapping of degree $d$, then $(1 / d) u \circ P$ is in $\mathcal{L}\left(\mathbb{C}^{n}\right)$.

Corollary 3.4 Let $P: \mathbb{C}^{n} \rightarrow \mathbb{P}^{m}, n \geq m$, be a non-degenerate polynomial mapping of degree $d$. Then there exists such a constant $k_{n}$ depending only on $n$ that

$$
\int_{\mathbb{C}^{n}}\left(\frac{1}{d} P^{*} \omega\right)^{j} \wedge \omega_{0}^{n-j} \leq k_{n}
$$

and if $X$ is the set where the rank of $J_{P}$ is less than $m$ and $W \in \mathbb{P}^{m} \backslash P(X)$, then

$$
\int_{\mathbb{C}^{n}}\left(\frac{1}{d} P^{*} \alpha_{w}\right)^{j} \wedge\left(\frac{1}{d} P^{*} \omega\right)^{m-1-j} \wedge \omega_{0}^{n-m+1} \leq k_{n},
$$

for $0 \leq j \leq m-1$.

The first statement follows directly from (3.5). To see why the second statement holds write $\left(3.6^{\prime}\right)$ for three functions: $v(z)=\log \left(1+|z|^{2}\right), u_{1}(z)=$ $(1 / d) \log |P(z)-W|^{2}, u_{2}(z)=(1 / d) \log |P(z)|^{2}$, and note that $L(v)=L\left(u_{2}\right)=\varnothing$ while $L\left(u_{1}\right)=\left\{P^{-1} W\right\}$. Since $W \notin P(X)$, we have $\operatorname{dim}\left\{P^{-1} W\right\}=n-m$, and by Corollary 3.1, $\left(d d^{c} u_{1}\right)^{k} \wedge\left(d d^{c} u_{2}\right)^{m-1-k} \wedge \omega_{0}^{n-m+1}$ is well defined for $k \leq m-1$, has locally finite mass, and therefore possesses a simple extension to $\mathbb{C}^{n}$. Hence (3.8) follows from $\left(3.6^{\prime}\right)$.

Lemma 3.5 Let $u$ and $v$ be locally bounded functions in $\mathcal{L}$ with $u=v+$ $O(1)$. Then

$$
\left|\left(\left(d d^{c} u\right)^{k}-\left(d d^{c} v\right)^{k}, \varphi\right)\right| \leq k \cdot C(u, v) \cdot C_{\varphi}
$$

where $C(u, v)=\sup |u-v|$.

Proof. Let $u$ and $v$ be locally bounded plurisubharmonic functions with $u=v+O(1)$ in a domain $\Omega \subset \mathbb{C}^{n}$. For $0 \leq m \leq n, 0 \leq k \leq m, 0 \leq j \leq k$, put $T_{k, m}^{j}=\left(d d^{c} u\right)^{j} \wedge\left(d d^{c} v\right)^{k-j} \wedge \omega_{0}^{m-k}$. We first prove that for each $\varphi \in$ $C_{0}^{\infty}(n-m, n-m)$,

$$
\left|\left(T_{k, m}^{j}, \varphi\right)-\left(T_{k, m}^{0}, \varphi\right)\right| \leq C(u, v) \cdot C(\varphi) \sum_{i=0}^{j-1} \int_{\Omega} T_{k-1, n}^{i} .
$$



Since

Put $h=u-v, R_{k, m}^{j}=d d^{c} h \wedge\left(d d^{c} u\right)^{j} \wedge\left(d d^{c} v\right)^{k-j} \wedge \omega_{0}^{m-k-1}, 0 \leq k \leq m-1$.

$$
T_{k, m}^{j}=T_{k, m}^{j-1}+R_{k-1, m}^{j-1}
$$

it follows that

$$
T_{k, m}^{j}-T_{k, m}^{0}=\sum_{i=0}^{j-1} R_{k-1, m}^{i}
$$

Now

$$
\begin{aligned}
\left|\left(R_{k-1, m}^{i}, \varphi\right)\right| & =\left|\left(h\left(d d^{c} u\right)^{i} \wedge\left(d d^{c} v\right)^{k-i-1} \wedge \omega_{0}^{m-k}, d d^{c} \varphi\right)\right| \\
& \leq C(u, v)\left|\left(\left(d d^{c} u\right)^{i} \wedge\left(d d^{c} v\right)^{k-i-1} \wedge \omega_{0}^{m-k}, d d^{c} \varphi\right)\right| \\
& \leq C(u, v) K_{\varphi} \int_{\Omega} T_{k-1, n}^{i}
\end{aligned}
$$

since $\left|d d^{c} \varphi\right| \leq K_{\varphi} \omega_{0}^{n-m+1}$.

It remains to note that, in the light of (3.5), the integrals of each of $T_{k, n}^{j}$ over $\mathbb{C}^{n}$ are all bounded by a constant. Hence (3.9) follows from (3.10).

Note that functions from $\mathcal{L}_{+}$are locally bounded by definition and hence do not charge pluripolar sets ([BT], Theorem 6.9). It follows that the pullbacks of all currents $\left(d d^{c} u\right)^{\ell}$ by non-degenerate mappings are well defined since the only difficulty is to extend the currents over the critical set that is pluripolar. Another way to see this is to approximate each such $u$ by a decreasing sequence $u_{k}$ of smooth plurisubharmonic functions. Then $\left(d d^{c} u\right)^{\ell}$ are also approximated by $\left(d d^{c} u_{k}\right)^{\ell}$, and it is enough to pass to the limit.

Corollary 3.6 Let $P: \mathbb{C}^{n} \rightarrow \mathbb{P}^{m}$ be a non-degenerate polynomial mapping of degree $d$, and let $U, V \in \mathcal{L}_{+}\left(\mathbb{P}^{m}\right)$. Then

$$
\left|\left(\left[\left(\frac{1}{d} P^{*} d d^{c} U\right)^{j}-\left(\frac{1}{d} P^{*} d d^{c} V\right)^{j}\right], \varphi\right)\right| \leq \frac{1}{d} C(U, V) \cdot C_{\varphi} .
$$

Proof. Put $u=(1 / d) U \circ P, v=(1 / d) V \circ P$, and use (3.9), taking into account that $C(u, v)=(1 / d) C(U, V)$. 
3.4. Extremal plurisubharmonic functions and pluripolar sets. A set $E$ in a domain $\Omega \subset \mathbb{C}^{n}$ is said to be pluripolar in $\Omega$ if there exists a function $u(z)$ plurisubharmonic in $\Omega$ such that $E \subset\{z: u(z)=-\infty\}$. Josefson has proved that locally pluripolar sets in $\mathbb{C}^{n}$ are globally pluripolar, i.e., that the function $u(z)$ in the definition may be chosen to be plurisubharmonic on entire $\mathbb{C}^{n}$. On the projective space there are no entire plurisubharmonic functions, and hence global pluripolarity makes no sense. So by pluripolar sets in $\mathbb{P}^{m}$ we will mean locally pluripolar sets or sets defined by plurisubharmonic sections.

If $\varphi$ is a function on $\mathbb{R}^{n}$, then its upper regularization $\varphi^{*}$ is defined as

$$
\varphi^{*}(x)=\limsup _{x^{\prime} \rightarrow x} \varphi\left(x^{\prime}\right) .
$$

Let $K$ be a compact set in $\mathbb{C}^{n}$. The extremal function (or the pluricomplex Green function with pole at infinity) of $K$ is defined as

$$
u_{K}=[\sup \{u(z): u \in \mathcal{L}, u \leq 0 \text { on } K\}]^{*} .
$$

The following properties of $u_{K}$ are well known (see, e.g., $[\mathrm{Kl}]$ ).

(1) if $K$ is pluripolar then $u_{K} \equiv \infty$.

(2) if $K$ is not pluripolar then $u_{k} \in \mathcal{L}_{+}$.

(3) if $K$ is not pluripolar then $\operatorname{supp}\left(d d^{c} u_{K}\right)^{n} \subset \partial K$.

From (3.3) it is clear that if $K$ is not pluripolar then $\mu=\left(d d^{c} u_{K}\right)^{n}$ is a probability measure on $\mathbb{C}^{n}$.

Lemma 3.7 Let $P: \mathbb{C}^{n} \rightarrow \mathbb{C}^{m}, n \geq m$, be a non-degenerate mapping of degree $d$, let $X$ be the set where rank $J_{P}<m$, let $K$ be a compact set in $\mathbb{C}^{m} \backslash P(X)$ and let $\mu=\left(d d^{c} u_{K}\right)^{m}$. Then:

$$
\int_{K}\left[P^{-1} W\right] d \mu=P^{*}\left(d d^{c} u_{K}\right)^{m}
$$

in the sense of currents.

Proof. Apply the left-hand side to a test form $\varphi$ :

$$
\begin{aligned}
\left(\int_{K}\left[P^{-1} W\right] d \mu, \varphi\right) & =\int_{K}\left(\left[P^{-1} W\right], \varphi\right) d \mu \\
& =\int_{\mathbb{C}^{m}}\left(\int_{P^{-1} W} \varphi\right)\left(d d^{c} u_{K}\right)^{m} \\
& =\left(\left(d d^{c} u_{K}\right)^{m}, \int_{P^{-1} W} \varphi\right)
\end{aligned}
$$


By the definition of a pullback,

$$
\left(\left(d d^{c} u_{K}\right)^{m}, \int_{P^{-1} W} \varphi\right)=\left(P^{*}\left(d d^{c} u_{K}\right)^{m}, \varphi\right) .
$$

The lemma is proved.

We would like to state a couple of simple auxilliary results to be used further on.

Lemma 3.8 Let $K$ be a set in $\mathbb{C}^{m}$ and let $\mu$ be a measure on $K$. Suppose that $K$ admits a representation of the form

$$
K=\bigcup_{j=1}^{\infty} \bigcap_{i=1}^{\infty} \bigcup_{k=i}^{\infty} E_{j}^{k},
$$

where the sets $E_{j}^{k}$ are such that

$$
\sum_{k=1}^{\infty} \mu\left(E_{j}^{k}\right)<\infty
$$

Then $\mu(K)=0$.

In particular, if $u_{K}$ is the extremal function of $K, \mu=\left(d d^{c} u_{K}\right)^{m}$ and (3.12)(3.13) hold, then $K$ is pluripolar.

Proof.

$$
\mu\left(\bigcup_{k=i}^{\infty} E_{j}^{k}\right) \leq \sum_{k=i}^{\infty} \mu\left(E_{j}^{k}\right) .
$$

The sum in the right-hand side is a tail of convergent series, so it tends to zero with $i$. This implies that

$$
\mu(K)=0 .
$$

If $K$ is not pluripolar, then $\mu=\left(d d^{c} u_{K}\right)^{m}$ is a probability measure on $K$. Hence the second statement follows.

Lemma 3.9 Let $\varepsilon_{k}$ be positive numbers, $\sum \varepsilon_{k}<\infty$. Let the functions $v, u_{1}, u_{2}, \ldots$ have the following properties:

(1) they are all non-negative;

(2) $v$ and $v-u_{j}$ are plurisubhamonic in a domain $Y \subset \mathbb{C}^{n}, \forall j$;

(3) $\int_{Y} u_{j} \omega_{0}^{n} \leq C, \forall j$ 
Then the set $E=\left\{z: \limsup _{j \rightarrow \infty} \varepsilon_{j} u_{j}>0\right\}$ is pluripolar in $Y$.

Proof. Let $E_{\delta}$ be the set

$$
E_{\delta}=\left\{z: \varepsilon_{j} u_{j} \geq \delta \text { for infinite number of indices } j\right\}
$$

Since

$$
E=\bigcup_{j=1}^{\infty} E_{1 / j}
$$

it is enough to prove that each $E_{\delta}$ is pluripolar. Put $S=\sum \varepsilon_{k}$ and

$$
U_{j}=S \cdot v-\sum_{k=1}^{j} \varepsilon_{k} u_{k}
$$

From our conditions it follows that $U_{j}$ are plurisubharmonic functions in $Y$ decreasing with $j$. Hence $U_{j} \searrow U$ and $U$ is either plurisubharmonic or identical $-\infty$.

To see that it is not identical $-\infty$ note that

$$
\int_{Y} U \omega_{0}^{n} \geq-\int_{Y} \sum_{k=1}^{\infty} \varepsilon_{k} u_{k} \omega_{0}^{n}=-\sum_{k=1}^{\infty} \varepsilon_{k} \int_{Y} u_{k} \omega_{0}^{n} \geq-S \cdot C>-\infty .
$$

Hence $U$ is plurisubhamonic, and if $z \in E_{\delta}$, then

$$
U(z)=S \cdot v(z)-\sum_{k=1}^{\infty} \varepsilon_{k} u_{k}(z) \leq S \cdot v(z)-\sum_{k=1}^{\infty} \delta=-\infty .
$$

The lemma is proved.

The following lemma uses an argument close to that of $[\mathrm{Fa}]$.

Lemma 3.10 Let $u_{1}, u_{2}, \ldots$ be a sequence of extremal plurisubhamonic functions of compact sets $K_{1}, K_{2}, \ldots$, respectively, all lying in the unit ball of $\mathbb{C}^{m}$ and let

$$
\sum \frac{1}{\max _{|z| \leq 1} u_{j}}<\infty
$$

Then the set

$$
E=\bigcap_{i=1}^{\infty} \bigcup_{j=i}^{\infty} K_{j}
$$

is pluripolar. 
Proof. By the extremality property, each $u_{j}$ vanishes on $K_{j}$ with a possible exception of a pluripolar set. It is no loss of generality to assume that $u_{j}$ vanishes on the entire $K_{j}$, since this will not affect the property of $E$ being pluripolar or non-pluripolar.

Denote by $M_{j}(r)$ the maximum of $u_{j}$ in the ball of radius $r$. Note that by (3.14) we have $M_{j}(1)>0$. In view of $(3.14)$ we can choose such a sequence $r_{j}>1$ of positive numbers increasing to infinity so slowly that

$$
\sum \frac{\log r_{j}}{M_{j}(1)}<\infty .
$$

Put

$$
v(z)=\sum_{j=1}^{\infty} \frac{u_{j}(z)-M_{j}\left(r_{j}\right)}{M_{j}(1)} .
$$

We claim that $v(z)$ is plurisubharmonic, $v(z)=-\infty, z \in E$, and $v$ is not identical $-\infty$.

Indeed, the terms in the series are plurisubharmonic functions, and if $|z|<$ $R$ then all the terms for which $r_{j}>R$ are negative, so the sum is either a plurisubharmonic function or identical $-\infty$. If $z \in E$, then there is an infinite sequence of numbers $j_{n}$ such that $z \in K_{j_{n}}$. By our assumption, this implies that $u_{j_{n}}(z)=0$. Hence, since $|z| \leq 1$ and all the terms in the series are negative,

$$
v(z) \leq \sum_{j_{n}}-\frac{M_{j_{n}}\left(r_{j_{n}}\right)}{M_{j_{n}}(1)}=-\infty .
$$

We would like to show that $v \not \equiv-\infty$.

Let $z_{j}$ be a point on the sphere $|z|=1$ where $u_{j}$ attains its maximum, $M_{j}(1)$. Fix a point $z_{0}$ on this sphere. Let $A_{j}$ be a unitary transformation taking $z_{0}$ to $z_{j}$. Consider an auxilliary function

$$
\widetilde{v}(z)=\sum_{j=1}^{\infty} \frac{u_{j}\left(T_{j} z\right)-M_{j}\left(r_{j}\right)}{M_{j}(1)} .
$$

Since $u_{j}\left(T_{j} z\right)$ are plurisubharmonic and have the same maximum on each sphere, $\widetilde{v}$ is plurisubharmonic for the same reason as $v$. Moreover, the functions $v$ and $\widetilde{v}$ have the same average value over each sphere, and hence if $v \equiv-\infty$ then $\widetilde{v}$ should have the same property. Clearly, we have

$$
\widetilde{v}\left(z_{0}\right)=\sum \frac{M_{j}(1)-M_{j}\left(r_{j}\right)}{M_{j}(1)} .
$$

Since $K_{j}$ lies in the unit ball, for which the extremal function is $\log ^{+}|z|^{2}$, we have $u_{j}(z)-M_{j}(1) \leq \log ^{+}|z|^{2}$, which implies that

$$
M_{j}(1)-M_{j}\left(r_{j}\right) \geq-\log r_{j}^{2} .
$$


Hence

$$
\widetilde{v}\left(z_{0}\right) \geq-\sum \frac{\log r_{j}^{2}}{M_{j}(1)}>-\infty
$$

by (3.15). The lemma is proved.

We remind the reader that two sequences or $(p, p)$-currents are called equidistributed with respect to a sequence $\left\{d_{k}\right\}$ if their difference divided by $d_{k}^{p}$ tends to the zero current.

In the proof of the next statement, the basic idea is to obtain two functions from $\mathcal{L}_{+}$by averaging over a non-pluripolar set.

Lemma 3.11 If $P_{k}$ is a lacunar sequence of non-degenerate polynomial mappings $\mathbb{C}^{n} \rightarrow \mathbb{P}^{m}, n \geq m$, deg $P_{k}=d_{k}$, then there exists a pluripolar set $E \in \mathbb{P}^{m}$ such that the sequences $\left[P^{-1} W\right]$ and $P^{*} \omega^{m}$ are equidistributed with respect to $\left\{d_{k}\right\}$ for $W \notin E$.

Proof. Let $\varphi$ be a test form. Put

$$
f_{k}(\varphi, W)=d_{k}^{-m}\left(\left(\left[P_{k}^{-1} W\right], \varphi\right)-\left(P_{k}^{*} \omega^{m}, \varphi\right)\right) .
$$

We first prove that $f_{k}(\varphi, W) \rightarrow 0$ outside a pluripolar set. Without loss of generality we may assume that $\varphi$ is chosen so that $f_{k}(\varphi, W) \in \mathbb{R}$.

Investigate the set

$$
\mathcal{E}(\varphi)=\left\{W \in \mathbb{P}^{m}: \lim _{k \rightarrow \infty} f_{k}(\varphi, W) \neq 0\right\}
$$

This set is a union of the set $\mathcal{E}^{+}$on which limsup $f_{k}>0$ and the set $\mathcal{E}^{-}$on which $\liminf f_{k}<0$. Thus it suffices to prove that either of these sets is pluripolar. We consider the first set since the other one is treated similarly.

Let

$$
\mathcal{E}_{\delta}=\left\{W \in \mathbb{P}^{m}: \limsup _{k \rightarrow \infty} f_{k}(\varphi, W)>\delta\right\} .
$$

It is enough to show that this set is pluripolar since

$$
\mathcal{E}^{+}=\bigcup_{j=1}^{\infty} \mathcal{E}_{1 / j}
$$

First consider the critical sets $X_{k}$ where rank $J_{P_{k}}<m$ and put $Y=$ $\bigcup_{k} P\left(X_{k}\right)$. Note that $Y$ is pluripolar and that the pullbacks of all the currents are well defined for $W \notin Y$. Suppose that the set $\mathcal{E}_{\delta}$ contains a non-pluripolar compact $K$. It suffices to suppose that $K$ lies inside the unit ball of the chart $\Omega_{0}$ and that $K \cap Y=\varnothing$. 
Let $E_{\delta}^{k}$ be the set of all $W \in K$ for which

$$
f_{k}(\varphi, W) \geq \delta .
$$

Since $f_{k}(\varphi, W)$ is a continuous function of $W$ outside $P\left(X_{k}\right)$, the sets $E_{\delta}^{k}$ are compact. Then

$$
K=\bigcap_{i=1}^{\infty} \bigcup_{k=i}^{\infty} E_{\delta}^{k}
$$

We would like to apply Lemma 3.10. Let $u_{k}$ be the extremal function of $E_{\delta}^{k}$ and let $M_{k}(1)=\sup _{|z| \leq 1} u_{k}$. We need to show that

$$
\sum \frac{1}{M_{k}(1)}<\infty
$$

Take the probability measure $\mu_{k}=\left(d d^{c} u_{k}\right)^{m}$. We have

$$
\begin{aligned}
\delta & \leq \int_{E_{\delta}^{k}} f_{k}(\varphi, W) d \mu(W) \\
& =\frac{1}{d_{k}^{m}} \int_{E_{\delta}^{k}}\left(\left(\left[P_{k}^{-1} W\right], \varphi\right)-\left(P_{k}^{*} \omega^{m}, \varphi\right)\right) d \mu .
\end{aligned}
$$

By Lemma 3.7

$$
\int_{E_{\delta}^{k}}\left[P_{k}^{-1} W\right] d \mu=P_{k}^{*}\left(d d^{c} u_{k}\right)^{m}
$$

and $P_{k}^{*} \omega^{m}$ does not depend on $W$. Hence the right-hand side of (3.16) equals

$$
\begin{aligned}
& \frac{1}{d_{k}^{m}}\left[\left(P_{k}^{*}\left(d d^{c} u_{k}\right)^{m}, \varphi\right)-\left(P_{k}^{*} \omega^{m}, \varphi\right)\right] \\
= & {\left[\left(\left(\frac{1}{d_{k}} P_{k}^{*} d d^{c} u_{k}\right)^{m}, \varphi\right)-\left(\left(\frac{1}{d_{k}} P_{k}^{*} \omega\right)^{m}, \varphi\right)\right] . }
\end{aligned}
$$

Put $v=\log \left(1+|z|^{2}\right)$. Note that since $u_{k}$ is an extremal function of a set in the unit ball, one has

$$
\log ^{+}|z|^{2} \leq u_{k}(z) \leq M_{k}(1)+\log ^{+}|z|^{2}
$$

so that

$$
\sup _{\mathbb{C}^{m}}\left|u_{k}(z)-v(z)\right| \leq M_{k}(1)+\log 2 .
$$

By Lemma 3.5 and Corollary 3.6 the right-hand side of (3.16) is bounded by

$$
\frac{1}{d_{k}} C_{\varphi} \cdot \sup \left|u_{k}-v\right| \leq \frac{1}{d_{k}} C_{\varphi}\left(M_{k}(1)+\log 2\right) .
$$


Hence

$$
\frac{1}{M_{k}(1)+\log 2} \leq \frac{C_{\varphi}}{\delta d_{k}} .
$$

In view of lacunarity, the right-hand side tends to zero with $k \rightarrow \infty$. Hence it is clear that $M_{k}(1)$ grows to infinity with $k$. So for $k$ large enough,

$$
\frac{1}{M_{k}(1)} \leq 2 \frac{C_{\varphi}}{\delta d_{k}},
$$

which yields (3.14). Thus by Lemma 3.10, $K$ is pluripolar and we arrive at a contradiction. Thus $\mathcal{E}(\varphi)$ is a pluripolar set. Now take a countable dense set of test forms $\left\{\varphi_{j}\right\}$ and put $\mathcal{E}=\bigcup \mathcal{E}\left(\varphi_{j}\right)$. Then $\mathcal{E}$ is also pluripolar.

We would like to show that outside of $\mathcal{E}$ we have equidistribution. Let $W \notin \mathcal{E}$. We need to show that $\left[P_{k}^{-1} W\right]$ is equidistributed with $P_{k}^{*} \omega^{m}$. Let $\varphi$ be an arbitrary test form. It suffices to show that for each $\varepsilon>0$, there exists such a number $k_{0}$ that

$$
d_{k}^{-m}\left(\left[P_{k}^{-1} W\right]-P_{k}^{*} \omega^{m}, \varphi\right)<\varepsilon, \quad \forall k>k_{0} .
$$

Choose a $j$ such that

$$
\left|\varphi_{j}-\varphi\right|<\frac{\varepsilon}{4 k_{n}} \omega^{n-m}
$$

Here $k_{n}$ is the constant in the right-hand side of (3.5). Once such a $j$ is chosen, we can find a $k_{0}$ such that, for any $k>k_{0}$,

$$
d_{k}^{-m}\left|\left(\left[P_{k}^{-1} W\right]-P_{k}^{*} \omega^{m}, \varphi_{j}\right)\right|<\frac{\varepsilon}{2},
$$

and hence

$$
\begin{aligned}
& \frac{\left|\left(\left[P_{k}^{-1} W\right]-P_{k}^{*} \omega^{m}, \varphi\right)\right|}{d_{k}^{m}} \\
\leq & \frac{\left|\left(\left[P_{k}^{-1} W\right]-P_{k}^{*} \omega^{m}, \varphi_{j}\right)\right|+\left|\left(\left[P_{k}^{-1} W\right]-P_{k}^{*} \omega^{m}, \varphi-\varphi_{j}\right)\right|}{d_{k}^{m}} \\
\leq & \frac{\varepsilon}{2}+\frac{\varepsilon}{4 k_{n}} \cdot \frac{\left|\left(\left[P_{k}^{-1} W\right]+P_{k}^{*} \omega^{m}, \omega^{n-m}\right)\right|}{d_{k}^{m}} \\
\leq & \varepsilon .
\end{aligned}
$$

The lemma is proved.

Remark 3.12 The following statement is a direct consequence of Corollary 3.6 (and a partial answer to a question in Section 2 of $[\mathrm{HP}]$ ):

Let $P_{k}: \mathbb{C}^{n} \rightarrow \mathbb{P}^{m}$ be a sequence of polynomial mappings with degrees $d_{k} \nearrow \infty$, and let $U, V \in \mathcal{L}_{+}$. Then the sequences of $(j, j)$-currents $P_{k}^{*}\left(d d^{c} U\right)^{j}$ and $P_{k}^{*}\left(d d^{c} V\right)^{j}$ are equidistributed with respect to $\left\{d_{k}\right\}$. 
3.5. The Poincaré-Lelong formula and properties of the proximity sequences. Let $f(z)$ be an entire function in $\mathbb{C}^{n}$. The Poincaré-Lelong formula relates the current $d d^{c} \log |f(z)|$ and the current of integration over the zero set $Z_{f}$ of $f(z)$ :

$$
d d^{c} \log |f(z)|=\left[Z_{f}\right] .
$$

If $f: \mathbb{C}^{n} \rightarrow \mathbb{C}^{m}, n \geq m$, is an entire mapping (vector function) and the zero set $Z_{f}$ has pure codimension $m$, then the Poincaré-Lelong formula for vector functions gives

$$
\left(d d^{c} \log |f(z)|\right)^{m}=\left[Z_{f}\right] .
$$

As an immediate consequence of the Poincaré-Lelong formula, one obtains the basic formula of the value distribution theory:

$$
d d^{c} \Lambda_{W}=\omega^{m}-[W] .
$$

If we preserve the notation $P_{k}^{*} \Lambda_{W}$ for the kernel of (1.1), we will be able to consider cases of codimension 1 and codimension $m$ in a unified manner.

We remind the reader that, for a sequence of non-degenerate polynomial mappings, the "proximity sequence" was defined as follows:

$$
m_{k}(W)=\int_{\mathbb{C}^{n}} P_{k}^{*} \Lambda_{W}(z) \wedge \omega_{0}^{n-\ell+1}, \quad \ell=\operatorname{codim} W .
$$

We would like to outline some properties of $m_{k}(W)$ as a function of $W$. Therefore, we omit the subscript $k$.

Let $\mu$ be the unit measure on $\mathbb{P}^{m}$ invariant with respect to unitary transformations. The measure $\mu$ in local coordinates is just $\omega^{m}$. It is well known (see, e.g., [Sh], Ch. 2, Section 4, Theorem 7) that, for some constant $c_{m}$ depending on $m$ only,

$$
\int_{\mathbb{P}^{m}} \Lambda_{\mathcal{W}}(Z) d \mu(\mathcal{W})=c_{m} \omega^{m-1}(Z)
$$

Therefore, if $d=\operatorname{deg} P, \ell=\operatorname{codim} W$, then

$$
\begin{aligned}
\frac{1}{d^{\ell-1}} \int_{\mathbb{P}^{m}} m_{P}(\mathcal{W}) d \mu(\mathcal{W}) & =\frac{1}{d^{\ell-1}} \int_{\mathbb{P}^{m}}\left(\int_{\mathbb{C}^{n}} P^{*} \Lambda_{\mathcal{W}} \wedge \omega_{0}^{n-\ell+1}\right) d \mu(\mathcal{W}) \\
& =c_{m} \int_{\mathbb{C}^{n}}\left(\frac{1}{d} P^{*} \omega\right)^{\ell-1} \wedge \omega_{0}^{n-\ell+1} .
\end{aligned}
$$

Hence, by (3.7),

$$
\frac{1}{d^{\ell-1}} \int_{\mathbb{P}^{m}} m_{P}(\mathcal{W}) d \mu(\mathcal{W}) \leq c_{m} \cdot k_{n}
$$


4. Proof of Theorem 1.1. Put

$$
g_{\mathcal{W}}(Z)=\log \frac{|W|^{2}|Z|^{2}}{|\langle W, Z\rangle|^{2}}
$$

By the Poincaré-Lelong formula,

$$
d d^{c} g_{\mathcal{W}}\left(P_{k}(z)\right)=P_{k}^{*} \omega-\left[P_{k}^{-1} \mathcal{W}\right],
$$

where $\left[P_{k}^{-1} \mathcal{W}\right]$ is the current of integration over the preimage of the hyperplane $\mathcal{W}$. For a test form $\varphi$, one gets

$$
\left|\left(P_{k}^{*} \omega-\left[P_{k}^{-1} \mathcal{W}\right], \varphi\right)\right|=\left|\left(g_{\mathcal{W}}\left(P_{k}(z)\right), d d^{c} \varphi\right)\right| \leq C_{\varphi} m_{k}(\mathcal{W}) .
$$

By definition of non-exceptional hyperplanes, this implies (i).

We remind the notion of $\alpha$-content. Let $\mathcal{U}$ be a countable or finite covering of a set $E \subset \mathbb{R}^{N}$ by balls $B_{j}$ of radii $R_{j}$. For $\alpha>0$, the $\alpha$-content $\lambda_{\alpha}$ is defined as

$$
\lambda_{\alpha}(E)=\inf \sum_{B_{j} \in \mathcal{U}} R_{j}^{\alpha},
$$

where the infimum is taken over all such coverings $\mathcal{U}$.

To prove (ii), we make use of the fact that $m_{k}$ are $\delta$-subharmonic functions of $W$ ( $m_{k}$ is an integrated difference of two logarithmic functions) with uniformly bounded Riesz charge and mean value, and of the following result:

Lemma 4.1 Let $u(x)$ be a $\delta$-subharmonic function on $\mathbb{P}^{k}$ with Riesz charge $\mu,\|\mu\|=1$, and let $\lambda_{\alpha}$ be the $\alpha$-content. Then, $\forall \varepsilon>0, \forall K>K_{0}$,

$$
\lambda_{2 k-2+\varepsilon}\left(\left\{X:\left|u(X)-c_{u}\right| \geq K\right\}\right) \leq \frac{C(k, \varepsilon)}{K^{1+\alpha}},
$$

where $c_{u}=\int_{\mathbb{P}^{k}} u(X) \omega^{k}(X), \alpha=\varepsilon /(2 k-2)$.

The lemma is easily reduced to the usual Cartan Lemma (see, e.g., [La], Ch. 3, Section 4, Corollary 1). Indeed, suppose that $\mu$ does not charge the projective hyperplane $X_{0}=0$. Then in the chart $X_{0} \neq 0$ considered as $\mathbb{R}^{2 k}$, we have

$$
u(x)=\int_{\mathbb{R}^{2 k}} \frac{d \mu(\xi)}{|x-\xi|^{2 k-2}}+c_{u}-\int_{\mathbb{R}^{2 k}} d \mu(\xi) \int_{\mathbb{R}^{2 k}} \frac{\omega_{0}^{k}(x)}{|x-\xi|^{2 k-2}},
$$

where we assume $X=[1, x], x \in \mathbb{R}^{2 k}$. Since

$$
0 \leq \int_{\mathbb{R}^{2 k}} \frac{\omega_{0}^{k}(x)}{|x-\xi|^{2 k-2}} \leq c(k),
$$

the modulus of the last term is bounded above by $c(k)$ and the assertion of the lemma is reduced to the estimation of the potential, i.e., to the Cartan Lemma. 
Denote by $E_{\delta}^{k}$ the set $\left\{W \in \mathbb{P}^{m}: m_{k}(\mathcal{W}) \geq \delta d_{k}\right\}$. For $k$ large enough, by Lemma 4.1 we get

$$
\lambda_{2 m-2+\varepsilon}\left(E_{\delta}^{k}\right) \leq \frac{C(k)}{\left(\delta d_{k}\right)^{1+\alpha}} .
$$

Writing the exceptional set $\mathcal{E}$ in the form

$$
\mathcal{E}=\bigcup_{n=1}^{\infty} \bigcap_{m=1}^{\infty} \bigcup_{k=m}^{\infty} E_{1 / n}^{k},
$$

and using the fact that

$$
\sum \frac{1}{d_{k}^{1+\alpha}}<\infty
$$

we conclude (see Lemma 3.8; formally, $\alpha$-content is not a usual measure; however, Lemma 3.8 uses in fact only subadditivity of the measure, so it holds in this case too) that $\lambda_{2 m-2+\varepsilon}(\mathcal{E})=0$. Hence $\mathcal{E}$ has Hausdorff dimension not exceeding $2 m-2$.

To prove (iii), we use $\delta$-plurisubharmonicity of $m_{k}$ and Lemma 3.9. Not losing generality, we may consider only one of the charts in $\mathbb{P}^{m}$. Suppose we are in $\Omega_{0}$ and $W=[1, w]$. Put

$$
v=\log \left(1+|w|^{2}\right), u_{k}=m_{k}([1, w]), k=1,2, \ldots, \varepsilon_{k}=\frac{1}{d_{k}},
$$

and verify the conditions of Lemma 3.9. The first condition is obvious, the second one follows from the definition of $m_{k}$. It remains to check that the average values

$$
\int_{\Omega_{0}} u_{k} \omega_{0}^{m}
$$

are uniformly bounded, which is exactly (3.18). Thus, we can apply Lemma 3.9 , which implies that the exceptional set $\mathcal{E}(\mathcal{P})$ is pluripolar. Theorem 1.1 is proved.

Remark 4.2 The relation (3.18) was derived from (3.17) which in this case actually looks like this:

$$
\int_{\mathbb{P} m} g_{\mathcal{W}}(Z) \omega^{m}(W)=C(m)\left(\stackrel{\text { def }}{=} \int_{\mathbb{C}^{m}} \log \left(1+|w|^{2}\right) \omega_{0}^{m}(w)\right),
$$

However, it is not necessary to consider average values over all of $\Omega_{0}$; by Lemma 3.9 we actually need to have some subset $A$ of $\mathbb{P}^{m}$ and some (positive) probability measure $\mu$ on it, such that

$$
\int_{A} g_{\mathcal{W}}(Z) d \mu(W)<C=C(A, \mu)<\infty .
$$


Existence of measures that have the property (4.2) was proved by H. Alexan$\operatorname{der}([\mathrm{A}]$, see also $[\mathrm{MSS}])$ :

Let $A \subset \mathbb{P}^{k}$ be a local analytic variety of positive dimension, which is not contained in any hyperplane. Then there exists a positive probability measure $\mu$ on $A$ with the property (4.2).

Thus we have, in fact, more precise information on the geometric structure of the exceptional set $\mathcal{E}$ : this set is pluripolar in each local analytic variety of positive dimension not contained in a hyperplane.

Remark 4.3 It is well known that studying preimages of algebraic divisors in $\mathbb{P}^{m}$ may be reduced to studying preimages of hyperplanes in a projective space of higher dimension. A natural parametrization space for algebraic divisors of degree $p$ is (via the Veronese mapping $V_{p}$ which maps $Z$ onto all homogeneous monomials in $Z_{0}, \ldots, Z_{m}$ of degree $\left.p\right) \mathbb{P}^{N}$ with $N=N(m, p) \stackrel{\text { def }}{=}\left(\begin{array}{c}m+p \\ p\end{array}\right)-1$. Each homogeneous polynomial $Q(Z)$ of degree $p$ is written as a sum

$$
Q(Z)=\sum_{i=0}^{N} W_{i} \cdot\left(V_{p}(Z)\right)_{i},
$$

and the element $W=\left[W_{0}, \ldots, W_{N}\right]$ corresponds to the divisor $\mathcal{W}=\{Z$ : $Q(Z)=0\}$. Since $\mathcal{W}$ may be identified with the corresponding hyperplane in $\mathbb{P}^{N}$, all the statements of Theorem 1.1 remain valid with $\omega$ replaced by $p \omega$.

Now we outline the way of obtaining the result for non-constant coefficients. This means that we allow the coefficients $W_{0}, \ldots, W_{N}$ of the homogeneous polynomial $Q(Z)$ defining the divisor $\mathcal{W}$ above depend polynomially on $z \in \mathbb{C}^{n}$. Thus, $Q \in K\left[Z_{0}, \ldots, Z_{m}\right]$ where $K=\mathbb{C}\left[z_{1}, \ldots, z_{n}\right]$, and we investigate the distribution of solutions of the equation $Q(z, P(z))=0$.

Let $Q(z, Z)$ be given. Then one can write it in the form

$$
Q(z, Z)=\sum_{i=0}^{N(m, p)} W_{i}(z)\left(V_{p}(Z)\right)_{i}
$$

Let $q$ be the maximal of degrees of $W_{i}$. Take a basis $\left\{e_{i}\right\}_{i=1}^{M(n, q)}$ of monomials in the space of polynomials of degree $q$ in $z_{1}, \ldots, z_{n}$. Clearly,

$$
M(n, q)=\sum_{p=0}^{q}(N(n, p)+1) .
$$

Now each of polynomials $W_{i}$ may be written in the form

$$
W_{i}(z)=\sum_{j=1}^{M(n, q)} W_{i j} \cdot e_{j} .
$$


The idea is to use auxilliary mappings $\widetilde{P}_{k}: \mathbb{C}^{n} \rightarrow \mathbb{P}^{M}$, where $M=$ $(N(m, p)+1) \cdot M(n, q)-1$. To define $\widetilde{P}$ we take $N(m, p)+1$ polynomials $\left(V_{p}(P(z))\right)_{i}$ and multiply each by $e_{j}, j=1, \ldots, M(n, q)$. Now

$$
Q(z, P(z))=\sum_{i=0}^{M} \widetilde{W}_{i} \widetilde{P}_{i}(z),
$$

where $\widetilde{W}$ is a vector of all $W_{i j}$. So we come to the case of hyperplanes in $\mathbb{P}^{M}$. It remains to note that

$$
|\widetilde{P}(z)|^{2}=\left|V_{p}(P(z))\right|^{2} \cdot \sum_{j=1}^{M}\left|e_{j}\right|^{2},
$$

and

$$
\begin{aligned}
\widetilde{g}(\mathcal{W} ; z) & \stackrel{\text { def }}{=} \log \frac{\left|V_{p}(P(z))\right|^{2} \sum\left|W_{i}\right|^{2}}{|Q(z, P(z))|^{2}} \\
& =\log \frac{|\widetilde{W}|^{2}|\widetilde{P}|^{2}}{|\langle\widetilde{W}, \widetilde{P}\rangle|^{2}}+\log \frac{\sum\left|W_{i}\right|^{2}}{|\widetilde{W}|^{2} \sum\left|e_{j}\right|^{2}} .
\end{aligned}
$$

Hence

$$
m_{k}(Q)=\widetilde{m}_{k}(\widetilde{W})+\frac{1}{d_{k}} \int_{\mathbb{C}^{n}} \log \frac{\sum\left|W_{i}\right|^{2}}{|\widetilde{W}|^{2} \sum\left|e_{j}\right|^{2}} \omega_{0}^{n}(z) .
$$

The last integral is obviously finite, and hence $m_{k}(Q)$ and

$$
\widetilde{m}_{k}(\widetilde{W})=\int_{\mathbb{C}^{n}} \widetilde{g}_{k}(\mathcal{W}) \omega_{0}^{n}(z)
$$

tend to zero simultaneously. All the statements of Theorem 1.1 hold automatically in this case as well.

We state below the most general form of Theorem 1.1. It is more convenient here to speak of exceptional polynomials rather than exceptional varieties. Also, denote $\left\{z: Q\left(z, P_{k}(z)\right)=0\right\}$ by $\mathcal{W}_{k}(Q)$.

Theorem 4.1 Let $\mathcal{P}$ be a sequence of polynomial mappings with increasing degrees. Then:

(1) for every non-exceptional polynomial $Q(z, Z)$ (homogeneous in $Z$ ) of degree $p$ in $Z$ and of degree $q$ in $z$, the sequences of currents $(1 / p)\left[\mathcal{W}_{k}(Q)\right]$ and $P_{k}^{*} \omega$ are $d_{k}$-equidistributed;

(2) the set $\mathcal{E}(\mathcal{P})$ of exceptional polynomials (considered as points of $\mathbb{P}^{M}, M=$ $M(p, m, q, n))$ has Hausdorff dimension not exceeding $2 M-2$;

(3) if the sequence $\mathcal{P}$ is lacunar, then $\mathcal{E}(\mathcal{P})$ is pluripolar in each local analytic variety of positive dimension in $\mathbb{P}^{M}$, which is not contained in a hyperplane. 
5. Proof of Theorem 1.2. First we exclude the (pluripolar) set $Y=$ $\bigcup_{k} P_{k}\left(X_{k}\right)$ of those values $W$ for which $P_{k}^{*} \Lambda_{W}$ may be not defined as a current in $\mathbb{C}^{n}$. Let $W \notin Y$. Applying $d d^{c}$ to $\Lambda_{W}$, we get, by the Poincaré-Lelong formula,

$$
d d^{c} \Lambda_{W}=\omega^{m}-[W] .
$$

Taking preimages under $P_{k}$, for a test $(n-m, n-m)$-form $\varphi$ one gets

$$
\left|\left(P_{k}^{*} \omega^{m}-\left[P_{k}^{-1} W\right], \varphi\right)\right|=\left|\left(P_{k}^{*} d d^{c} \Lambda_{W}, \varphi\right)\right|=\left|\left(P_{k}^{*} \Lambda_{W}, d d^{c} \varphi\right)\right| \leq C_{\varphi} m_{k}(W) .
$$

By the definition of non-exceptional points, this implies (i).

Suppose that $W=[1, w]$ and $Z=[1, z]$ are points in $\Omega_{0}$. To prove (ii) we will use the following estimate due to J. Carlson [Ca]:

for every $\varepsilon>0$ there exists a constant $C_{\varepsilon}$, such that

$$
\Lambda_{W}(Z) \leq C_{\varepsilon} K_{2 m-2+\varepsilon}(W, Z) \omega^{m-1}(Z),
$$

where $K_{\alpha}(W, Z)=1 /\left(|w-z|^{\alpha}\right)$.

We remind the reader that $K_{\alpha}(W, Z)$ is a kernel function for $\alpha$-potential (we refer to [La] for facts concerning potential theory in $\mathbb{R}^{N}$ which are used below). Let $\Omega$ be a compact set of positive $\alpha$-capacity in $\Omega_{0}, \quad \alpha=2 m-2+\varepsilon, \varepsilon>$ $0, \Omega \cap Y=\varnothing$. Denote the equilibrium $\alpha$-measure for $\Omega$ by $\mu_{\alpha}$, the corresponding potential by $V_{\alpha}$ and $\alpha$-capacity by $c_{\alpha}$. Integrate our function $m_{k}(W)$ with respect to $\mu_{\alpha}$ over $\Omega$ :

$$
\begin{aligned}
\int_{\Omega} m_{k}(W) d \mu_{\alpha}(W) & =\int_{\Omega}\left(\int_{\mathbb{C}^{n}} P_{k}^{*} \Lambda_{W} \wedge \omega_{0}^{n+1-m}(z)\right) d \mu_{\alpha}(W) \\
& =\int_{\mathbb{C}^{n}}\left(P_{k}^{*} \int_{\Omega} \Lambda_{W} d \mu_{\alpha}(W)\right) \wedge \omega_{0}^{n+1-m}(z) \\
& \leq C_{\varepsilon} \int_{\mathbb{C}^{n}} P_{k}^{*}\left(\int_{\Omega} K_{\alpha}(W, Z) d \mu_{\alpha}(W) \omega^{m-1}(Z)\right) \\
& \leq C_{\varepsilon} \int_{\mathbb{C}^{n}} P_{k}^{*}\left(V_{\alpha}(Z) \omega^{m-1}(Z)\right) \wedge \omega_{0}^{n+1-m}(z) \\
& \leq \frac{C_{\varepsilon}}{c_{\alpha}(\Omega)} \int_{\mathbb{C}^{n}} P_{k}^{*} \omega^{m-1}(Z) \wedge \omega_{0}^{n+1-m}(z) .
\end{aligned}
$$

We claim that the right-hand side may be estimated by $C /\left(c_{\alpha}(\Omega)\right) \cdot d_{k}^{m-1}$ with some constant $C$.

Indeed, by (3.7), for all $0 \leq j \leq m$,

$$
\int_{\mathbb{C}^{n}}\left(\frac{1}{d_{k}} P_{k}^{*} \omega\right)^{j} \wedge \omega_{0}^{n-j} \leq k_{n} .
$$


Therefore,

$$
\int_{\Omega} m_{k}(W) d \mu_{\alpha}(W) \leq \frac{C_{\varepsilon}^{\prime}}{c_{\alpha}(\Omega)} d_{k}^{m-1} .
$$

Now suppose that the exceptional set $\mathcal{E}$ has Hausdorff dimension $\alpha_{0}>2 m-2$. Take a compact set $K \subset \mathcal{E} \cap \Omega_{0}$ of Hausdorff dimension $\alpha_{0}$ and such a positive number $\varepsilon$ that $\alpha=2 m-2+\varepsilon<\alpha_{0}-\varepsilon$. Denote by $E_{\delta}^{k}$ the set $\{W \in K$ : $\left.m_{k}(W) \geq \delta d_{k}^{m}\right\}$, take a compact set $K_{\delta}^{k} \subset E_{\delta}^{k}$ whose $\alpha$-capacity is larger than $1 / 2 c_{\alpha}\left(E_{\delta}^{k}\right)$, and denote by $\mu_{\alpha}$ its equilibrium $\alpha$-measure. The estimates above give

$$
c_{\alpha}\left(E_{\delta}^{k}\right) \leq 2 c_{\alpha}\left(K_{\delta}^{k}\right) \frac{2 C_{\varepsilon}^{\prime} d_{k}^{m-1}}{\int_{K_{\delta}^{k}} m_{k}(W) d \mu_{\alpha}(W)} \leq \frac{C_{\varepsilon}^{\prime}}{\delta d_{k}},
$$

and thus, by Corollary 2 of [La], ch. 3, Section 4, we obtain an estimate of $(\alpha+\varepsilon)$-content:

$$
\lambda_{\alpha+\varepsilon}\left(E_{\delta}^{k}\right) \leq\left(\frac{C^{\prime}}{\delta d_{k}}\right)^{1+\beta}
$$

for some $\beta>0$.

Since

$$
\sum \frac{1}{d_{k}^{1+\beta}}<\infty
$$

and $K$ may be written in the form

$$
K=\bigcup_{n=1}^{\infty} \bigcap_{m=1}^{\infty} \bigcup_{k=m}^{\infty} E_{1 / n}^{k}
$$

we see, by Lemma 3.8 , that $\lambda_{2 m-2+2 \varepsilon}(K)=0$. Since $2 m-2+2 \varepsilon<\alpha_{0}$, we arrive at a contradiction, which proves statement (ii). Statement (iii) is exactly Lemma 3.11. The theorem is proved.

5.1. Acknowledgements. We would like to thank L. Gruman, N. Sibony, L. I. Ronkin and B. A. Taylor for fruitful discussions. We are especially thankful to S. Yu. Favorov whose valuable remarks helped to improve many places in the exposition. We also thank the referee for drawing our attention to certain inaccuracies in the earlier version of the paper. The first version of the paper was written while the first author was visiting the University of Düsseldorf and the University of Toulouse being supported by The Alexander von Humboldt Foundation, and the second author was visiting Purdue University. We wish to express our gratitude to the organizations above for their support and hospitality.

\section{REFERENCES}

[A] H. Alexander. A note on projective capacity. Canad. J. Math. 34 (1982), $1319-1329$. 
[BS1] E. Bedford, J. Smillie. Polynomial diffeomorphisms of $\mathbb{C}^{2}$ : currents, equilibrium measure and hyperbolicity. Invent. Math. 87 (1990), 69-99.

[BS2] E. Bedford, J. Smillie. Polynomial diffeomorphisms of $\mathbb{C}^{2}$ : stable manifolds and recurrence. J. of Amer. Math. Soc. 4 (1991), 4, 657-679.

[BT] E. Bedford, B. A. TAYLOR. A new capacity for plurisubharmonic functions. Acta Math. 149 (1982), 1-40.

[Br] H. Brolin. Invariant sets under iteration of rational functions. Ark. Math. 61 (1965), 103-144.

[Ca] J. Carlson. A moving lemma for the transcendental Bezout problem. Annals of Math. 103 (1976), 305-330.

[De] J.-P. Demailly. Monge-Ampère operators, Lelong numbers and intersection theory. Complex Analysis and Geometry, Univ. Series in Math., V.Ancona \& A.Silva ed., Plenum Press, New-York, 1993, 115-193.

[EL] A. E. Eremenko, M. Yu Lyubich. Dynamics of analytic transformations. Leningrad J. Math. 1 (1989), 1-70.

[ES] A. E. ERemenko, M. L. Sodin. Iterations of rational functions and distribution of values of the Poincaré function. Function Theory, Funct.Anal. and their Appl. 53 (1990), 18-26 (Russian); Engl. transl. in J. of Soviet Math. 58 (1992), 504-509.

[Fa] S. Yu. Favorov. On the growth of plurisubharmonic functions. Siber. Math. J. 34 (1983), n 1, 168-174.

[FS] J. E. Fornaess, N. Sibony. Complex Henon mappings in $C^{2}$ and FatouBieberbach domains. Duke Math. J. 65 (1992), 345-380.

[FLM] A. Freire, A. Lopes, R. MAÑé. An invariant measure for rational maps. Bol. Soc. Bras. Mat. 6 (1983), 45-62.

[Gr] L. Gruman. Ensembles exceptionneles pour les applications holomorphes dans $\mathbb{C}^{n}$. Séminaire P. Lelong-P. Dolbeault-H. Skoda, 1981-1983, Lecture Notes 1028, 125-162.

[HP] J. H. Hubbard, P. Papadopol. Superattractive fixed points in $\mathbb{C}^{n}$. Preprint.

[Kl] M. Klimek.Pluripotential theory. Oxford University Press, 1991.

[La] N. S. Landkof. Foundations of Modern Potential Theory. Springer-Verlag, Berlin, 1972.

[LG] P. Lelong, L. Gruman.Entire functions of several complex variables. Springer-Verlag, Berlin, 1986.

[L] M. Yu. Lyubich. Entropy properties of rational endomorphisms of the Riemann sphere. Ergodic theory and dynamical systems 3 (1983), 351-385.

[MSS] R. Molzon, B. Shiffman, N. Sibony. Average growth estimates for hyperplane sections of entire analytic sets. Math. Ann. 257 (1981), 43-59.

[Sk] H. Skoda. Prolongement des courants positifs fermés de masse fini. Invent. Math. 66 (1982), 361-367.

[So] M. L. Sodin. Value distribution of sequences of rational mappings. Advances in Soviet Math., 11, Entire and Subharmonic Functions, ed. by B. Ya. Levin, 1992.

[Sh] B. V. Shabat. Distribution of values of holomorphic mappings. Amer. Math. Soc., Providence, R.I., 1985.

[T] B. A. TAYLOR. An estimate for an extremal plurisubharmonic function on $\mathbb{C}^{n}$. Séminaire P. Lelong-P. Dolbeault-H. Skoda, 1981-1983, Lecture Notes 1028, $318-328$.

\section{Mikhail Sodin}

Theory of Functions Department

Institute for Low Temperature Physics and Engineering

Academy of Science of Ukraine 
47 Lenin Avenue

310164 Kharkov, Ukraine

Alex Russakovskil

Department of Mathematics

John Hopkins University

Baltimore, Maryland 21218

Received: January 13th, 1993; revised: May 17th, 1995. 